\title{
Articles
}

Rapid communication

\section{Recording of subcutaneous glucose dynamics by a viscometric affinity sensor}

\author{
U. Beyer ${ }^{1}$, D. Schäfer ${ }^{1}$, A. Thomas ${ }^{1}$, H. Aulich ${ }^{1}$, U.Haueter ${ }^{2}$, B. Reihl ${ }^{2}$, R. Ehwald ${ }^{3}$ \\ ${ }^{1}$ Disetronic Medical Systems GmbH, Sulzbach/Ts., Germany \\ ${ }^{2}$ Disetronic Medical Systems AG, Burgdorf, Switzerland \\ ${ }^{3}$ Humboldt-University Berlin, Germany
}

\begin{abstract}
Aims/hypothesis. To provide a nonenzymatic sensor for glucose monitoring in subcutaneous tissue.

Methods. A continuously working affinity sensor based on the glucose-dependent viscosity of a sensitive liquid containing dextran and concanavalin A has been designed by arranging a microdialysis probe, two flow-resisting capillaries and two pressure transducers in a linear flow system. It allows synchronous processing of the viscosity of the sensitive liquid at the standard glucose concentration and the glucose concentration to be measured. In preliminary human trials the sensor was implanted into the subcutaneous tissue of the forearm and its read-out was compared with capillary blood concentrations.

Results. In vitro, the viscometric sensor shows a linear and long-term stable dependence on the glucose concentration without detectable drift. At the applied flow rate of the sensitive liquid (about $5 \mu \mathrm{l} / \mathrm{h}$ ) the
\end{abstract}

technical delay is 5 to $10 \mathrm{~min}$. The read-out of the implanted sensor followed the dynamics of the capillary blood glucose concentrations with a time-shift of 10 to $15 \mathrm{~min}$ but showed a systematic error when based on precalibration with polymer-free glucose solutions. After appropriate in vivo calibration, the readout was in good or acceptable coincidence with capillary blood concentrations according to the error grid method and did not show any detectable reduction of sensitivity during the periods of measurement (up to $44 \mathrm{~h}$ ).

Conclusion/interpretion. The viscometric-affinity sensor is an efficient tool for current research on glucose monitoring in the subcutaneous tissue and can potentially be further developed for routine clinical use. [Diabetologia (2001) 44: 416-423]

Keywords Concanavalin A, dextran, glucose monitoring, microdialysis probe, subcutaneous tissue, viscometric affinity sensor.
Portable glucose sensors with an accurate and longterm stable in vivo function have been in demand for several decades because such devices are of strategic importance in the diagnosis and treatment of diabetes mellitus [1-3]. Despite remarkable progress, the

Received: 24 July 2000 and in final revised form: 17 November 2000

Corresponding author: Dr. R. Ehwald, Humboldt-University Berlin, Faculty of Mathematics and Natural Science I, Department of Biology, Invalidenstraße 42, 10115 Berlin, Germany Abbreviations: ConA, Concanavalin A; GOD, glucose oxidase; $F_{n}$, relative fluidity; SL, sensitive liquid; VAS, viscometric-affinity sensor. development of new and improvement of existing prototypes is desirable.

Efforts have focused on transdermal glucose sensors because the glucose concentrations of capillary blood and interstitial liquid of the subcutaneous tissue are closely related [4-8]. These sensors measure the glucose concentration either after electroosmotic transfer of the interstitial liquid to the skin surface [9] or by a microsensor implanted into the subcutaneous tissue. Most work has been carried out using needle-like transdermal amperometric sensors with immobilised glucose oxidase (GOD) [10-18]. To render the highly sensitive and oxygen-dependent enzyme reaction glucose-limited, the immobilised enzyme is usually covered by a membrane that is less 
permeable to glucose than to dissolved oxygen [13-16]. Adapting the sensing process to the microenvironment in the subcutaneous tissue was found to be difficult because implantation results in unforeseeable decreases in sensor sensitivity. Tissue-generated increases in diffusion resistances or the presence of electrochemically active solutes have been suggested as possible reasons $[2,8,16,17]$.

Some of these difficulties have been overcome in the development of the GOD-microsensor supplied by Minimed (Sylmar, Calif., USA), which obtained a pre-market approval last year [18]. The application of this sensor requires individual and frequent calibrations on the basis of parallel capillary blood analysis and, therefore, further research on glucose monitoring in the subcutaneous tissue is important. One approach is the use of electrochemical GOD-microsensors in combination with microdialysis because sensors are more stable in the dialysate than in direct contact with tissue [19-22].

Microdialysis also enables the construction of implantable glucose sensors based on a competitive affinity assay [20]. In the affinity sensors [23], the carbohydrate binding protein concanavalin A (ConA), a lectin of plant origin, and its polymer glycoligand, dextran, are enclosed in an implantable dialysis fibre segment. Unlike the polymers, glucose can diffuse through the fibre membrane. Binding of ConA to dextran is reversibly weakened by glucose because the ConA-binding sites have an affinity to both the free sugar and the terminal glucose residues of the polysaccharide. The original prototype and further variants of the glucose-affinity sensor use optoelectronics to measure a glucose-dependent fluorescence signal created in the fibre lumen either by sorption/ desorption of fluorescence-labelled polymer molecules or by association-dependent fluorescence quenching [23-27].

Viscometric affinity assays for glucose and related sensor concepts [28-30] use the high and glucose-dependent viscosity found in concentrated solutions of high-molecular-weight dextran and ConA. Saturating concentrations of free glucose reduce this viscosity by more than one order of magnitude [28]. By using a suitable reference measurement, the viscometric glucose assay is insensitive to variations in measuring conditions such as temperature and polymer concentration [31]. Although different concepts of viscometric sensors have been developed [29, 32-33], applicable miniaturised prototypes for continuous measurement in the subcutaneous tissue have not been available before. We describe such a device and report on its function in vitro and in preliminary human trials.

\section{Material and methods}

Sensitive liquid. The sensitive liquid (SL) contained $7 \%$ Dextran T2000 (Pharmacia, Uppsala, Sweden), 0.7\% concanavalin A (type IV, cat. no. C 2010, Sigma-Aldrich Chemie, Deisenhofen, Germany), $5 \mathrm{mmol} / \mathrm{l}$ D-glucose, $0.1 \%$ phenol and $0.1 \% \mathrm{~m}$ cresol. These substances were dissolved in a physiological saline solution. The $\mathrm{pH}$ was adjusted to 7.4.

Glucose solutions. Glucose solutions used for calibration and in vitro tests containted $130 \mathrm{mmol} / \mathrm{l} \mathrm{NaCl}, 5 \mathrm{mmol} / \mathrm{l} \mathrm{KCl}$, $15 \mathrm{mmol} / 1 \mathrm{NaN}_{3}$, and $10 \mathrm{mmol} / 1 \mathrm{TRIS}$-(hydroxmethyl)-aminomethane-hydrochloride at $\mathrm{pH}$ 7.4.

Preanalysed blood plasma samples. A series of 20 coded clinical blood plasma samples (volume $1 \mathrm{ml}$ ) was obtained from Dr. A. Kage, Humboldt-University Berlin, Medical Faculty (Charitè), Institute of Laboratory Medicine and Pathobiochemistry. The glucose concentration of these samples had been measured by a Hitachi 717 analyser (Diagnostic Chemicals, Prince Edward Island, Canada) using the hexokinase/ glucose-6-phosphate-dehydrogenase method (Boehringer Mannheim, Mannheim, Germany) with a relative error below $2 \%$. Samples were stored frozen until use.

Measurement of glucose concentration in capillary blood. At each sampling, the glucose concentration in the capillary blood was calculated as the mean of two values obtained by parallel measurements with two different Accutrend sensors (Boehringer Mannheim) or Glucometer Elite 2000 (Bayer Leverkusen, München, Germany).

Microdialysis fibre. The previously applied microdialysis fibre [30] with an inner diameter of $200 \mu \mathrm{m}$ (Kunstseidewerk Pirna, Germany) was used. The membrane of this fibre consists of regenerated cellulose and is completely impermeable to molecules with a Stokes' radius greater than $2 \mathrm{~nm}$ (proteins with a molecular weight $>15,000 \mathrm{M}_{\mathrm{r}}$ ) in long-term experiments [34].

Viscometric sensor. The sensor measures the viscosity of the SL before and after its transfer through a dialysis fibre [32]. It includes a dialysis probe, flow-resisting capillaries and pressure transducers (Fig. 1). The SL is continuously pumped with low rate $(\sim 5 \mu \mathrm{l} / \mathrm{h})$ through the system. The pressure transducers $\mathrm{P} 1$ and P2 (AktivSensor GmbH, Stahnsdorf, Germany) measure the flow-mediated pressures before $\left(p_{1}\right)$ and after $\left(p_{m}\right)$ the reference capillary $\left(\mathrm{R}_{\mathrm{ref}}\right)$. The pressure difference created by the flow through $\mathrm{R}_{\text {ref }}\left(p_{r e f}=p_{1}-p_{m}\right)$ is a measure of the viscosity of the unmodified SL with the standard glucose concentration $(5 \mathrm{mmol} / \mathrm{l})$. Because the SL reaches the measuring capillary $\left(R_{m}\right)$ after exchange with the external matrix (buffered solutions, subcutaneous tissue), $p_{m}$ is proportional to the viscosity of the SL at the glucose concentration to be measured.

The proprietary implantable needle-like transdermal dialysis probe consists of two parallel steel cannulae (length $30 \mathrm{~mm}$, outer diameter each $0.6 \mathrm{~mm}$ ) with a sharpened tip. The inlet cannula is sealed at the tip and its lumen is connected with the dialysis fibre in the apical region. The outlet capillary surrounds the major part of the dialysis fibre segment. The membrane of the latter is exposed to the external liquid or the subcutaneous tissue at two parallel lateral windows of $11-\mathrm{mm}$ length. At its outlet, the dialysis fibre segment is directly connected with the measuring capillary. The pressure transducers, the reference capillary, and a waste container are integrated into a flat rectangular box $(13 \times 35 \times 47 \mathrm{~mm}$, about $25 \mathrm{~g})$. Flexible tubes connect this box with the dialysis probe and a modified H-Tron V100plus pump (Disetronic Medical Systems, 
A

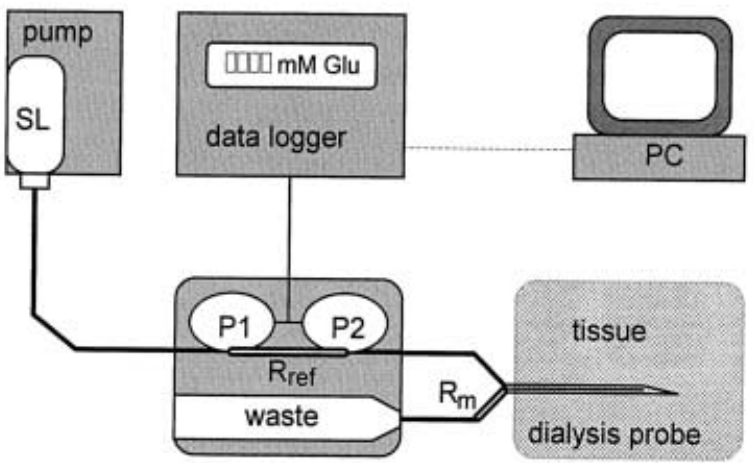

B

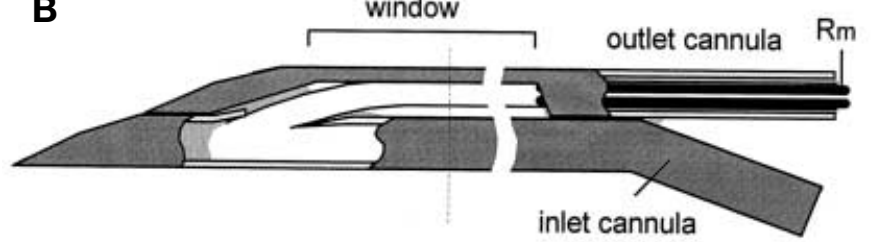

window

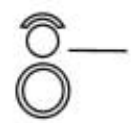

dialysis fiber

Fig.1A, B. On-line sensor for continuous glucose monitoring. A Scheme of the entire measuring device. The SL is pumped through flow-resisting capillaries $\left(R_{\text {ref }}, R_{m}\right)$ and a dialysis probe into a waste container. Its viscosity before and after flow through the dialysis probe is derived from signals of the pressure transducers $\mathrm{P} 1$ and $\mathrm{P} 2$. The data logger stores these signals and transforms them to relative fluidity $\left(F_{r}\right)$ and glucose concentration. Data transfer to the computer enables in vivo calibration. B Dialysis probe (not drawn true to scale)

Sulzbach, Germany), which supplies the sensor with a nearly constant and pulse-free flow (usually $5 \mu \mathrm{l} / \mathrm{h}$ ) of fresh SL. A programmable data logger (TT5F, Onset Computer, Pocasset, Mass., USA) was used for signal transformation and storage as well as display of glucose concentrations and data download to a personal computer.

Transformation of the viscosity-related signals into glucose concentrations. The relative fluidity $\left(F_{r}\right)$ of the sensitive liquid, which shows a linear dependence on the glucose concentration $\mathrm{C}$ [31], is obtained from the signals given by pressure transducer $1\left(p_{1}\right)$ and pressure transducer $2\left(p_{m}\right)$ by:

$$
F_{r}=\frac{p_{l}-p_{m}}{p_{m}+\alpha \cdot\left(p_{l}-p_{m}\right)}
$$

wherein $\alpha$ represents a linearization coefficient, $\left(p_{1}-p_{m}\right)$ is related to the viscosity of the SL at the reference glucose concentration $(5 \mathrm{mmol} / \mathrm{l})$ and $p_{m}$ to the viscosity of the SL at the measuring glucose concentration. Because flow resistances of the reference and measuring capillaries $\left(R_{\text {ref }}\right.$ and $\left.R_{m}\right)$ were neither equal nor accurately defined, the appropriate value of $\alpha$ (enabling the maximum correlation coefficient of the linear regression between $F_{r}$ and the glucose concentration) had to be calculated for each combination of the measuring system and dialysis probe. To continuously display and register glucose concentrations the pressure signals that were actually measured were on-line transformed by the data logger to $F_{r}$ and the glucose concentration every $30 \mathrm{~s}$. After read-out to the computer, stored values of six subsequent measurements were averaged by a macro in the Excel program to obtain means of 3- min intervals which were used for further evaluations and graphics.

Implantation of the dialysis probe and wearing of the viscometric affinity sensor $(V A S)$. After disinfecting the skin surface with a Cutasept F spray (Bode Chemie, Hamburg, Germany), the precalibrated dialysis probe was implanted in the subcutaneous tissue of the left forearm (inner face) parallel to the skin's surface without interruption of the flow. The box with the pressure transducers was fixed on the skin by an adhesive tape. The pump was fastened by elastic ribbons to the forearm close to the measuring box and the logger was worn on the belt with the signal cable routed through the shirt sleeve. The system on the forearm was covered with an elastic wool tissue to prevent temperature fluctuations.

Calibration by capillary blood values. Stored pressure signals were transferred to the computer for a preliminary in vivo calibration on the basis of at least five measurements of capillary blood glucose concentrations $\left(C_{c a p}\right)$ obtained within about $3 \mathrm{~h}$ after an initial adaptation period of 0.5 to $1.5 \mathrm{~h}$ after implantation. Programming of the logger with the preliminary calibration parameters gave reliable values on the display during the experiment. For the more accurate retrospective calibration, the full set of measured capillary blood values at times $\mathrm{t}\left(C_{\mathrm{cap}}^{t}\right)$ was correlated with corresponding delayed $F_{r}$ values $\left(F_{r}^{\mathrm{t}+\tau}\right)$ using different estimates of the time-shift $\tau$, and the best-fitting value (maximum correlation coefficient) was obtained by approximation. The linear regression obtained with this $\tau$ value was used for calibration.

\section{Results}

Kinetics and stability of the sensor response. When the dialysis probe of the sensor was transferred from one glucose standard solution to the next (Fig.2), the read-out reached a new constant value within a time of 5 to $10 \mathrm{~min}$. As the glucose value displayed is obtained by reference to the internal standard, it did not show any drift. At all tested concentrations the read-out was stable for at least 14 days, and its dependence on the glucose concentration within the physiologically interesting range is approximately linear (Fig. 2). An increase of the flow rate decreased the response time but did not reduce the sensor read-out, unless this rate exceeded $15 \mu \mathrm{l} / \mathrm{h}$ (data not shown). To ensure $100 \%$ recovery in the subcutaneous tissue even when external diffusion resistance increased, a flow rate of approximately $5 \mu \mathrm{l} / \mathrm{h}$ was adjusted for in vivo and in vitro experiments.

Preliminary human trials. In self-tests carried out by some of the authors the dialysis probe was implanted into the subcutaneous tissue of the forearm and the sensor response was registered. No inflammatory reactions of the skin were observed. Generally the relative fluidity $F_{r}$ obtained by the sensor in the subcutaneous tissue varied in correlation with the measured capillary blood concentration (Table 1) but the glucose concentrations computed by parameters of in vitro calibration were significantly $(\alpha=0.01)$ below 
A
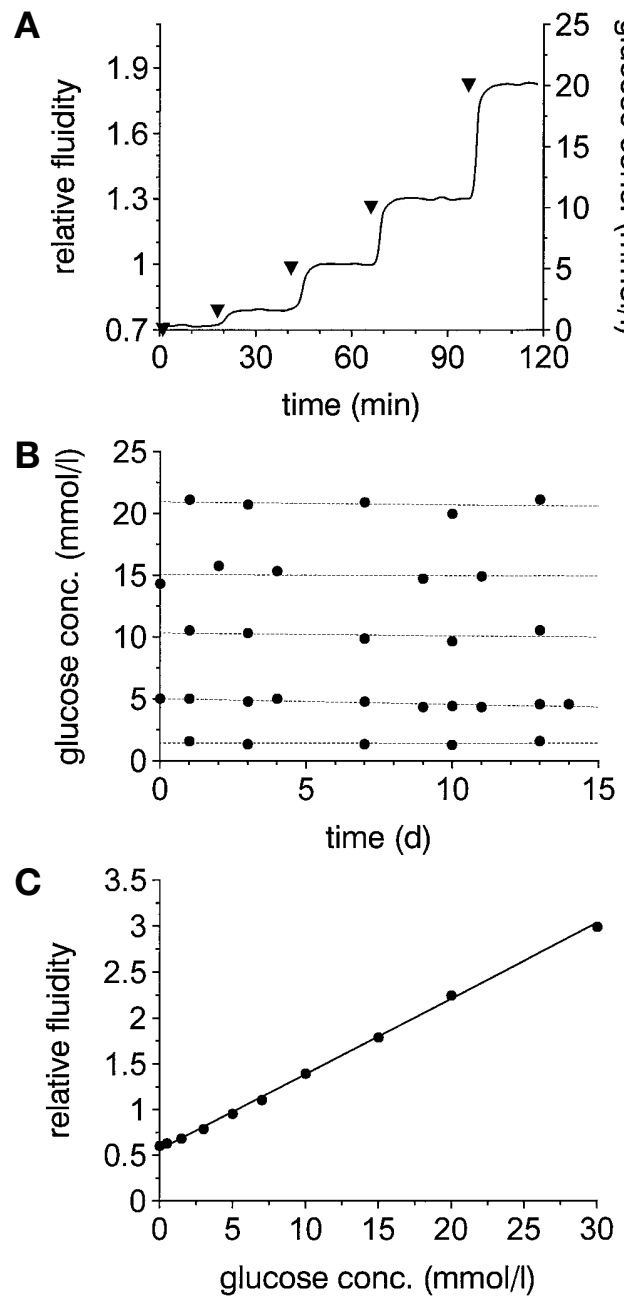

Fig. 2 A-C. Kinetics, sensitivity and stability of the VAS. A The dialysis probe was transferred to standard solutions containing glucose in concentrations of $0,1.5,5,10$ or $20 \mathrm{mmol} / \mathrm{l}$ (left to right) at times indicated by arrows. The additional ordinate shows the glucose concentration displayed by the calibrated sensor. B Glucose concentration indicated by another calibrated sensor at repeated measurements with standard solutions containing glucose in concentrations of $1.5,5,10,15$ and $20 \mathrm{mmol} / \mathrm{l}$. C Linear regression of glucose concentration and $F_{r}$. Points represent mean values of five replications measured in intervals of 2 or 3 days as indicated in $\mathbf{B}$. Data include those shown in $\mathbf{B}$ and measurements with further standard solutions. $r^{2}=0.996$, highest relative standard deviation: $4.7 \%$

the capillary blood glucose concentrations. Likewise, in blood plasma samples the glucose concentrations read out by the precalibrated sensor were lower than accurately predetermined values. In both cases, the mean difference is 2 to $3 \mathrm{mmol} / \mathrm{l}$ (Fig. 3 ).

Because in vitro calibration was insufficient, the sensor read-out was based on a calibration by the capillary blood values as described in Material and methods. Results of all experiments carried out for measuring periods of more than $12 \mathrm{~h}$ are summarised in Table 1. Subjects 1-3 (curves not shown) had normal dynamics of capillary blood values with a slight
A

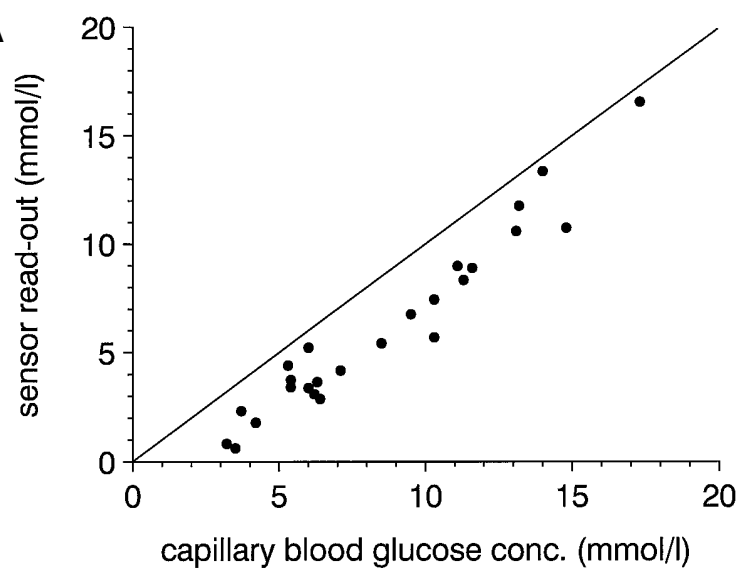

B

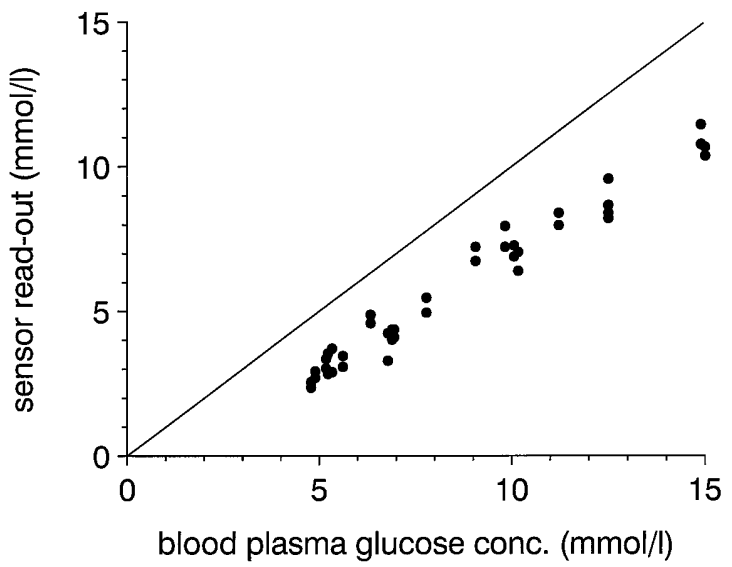

Fig.3 A, B. Read-out of precalibrated sensors in the subcutaneous tissue and in blood plasma. Sensor read-out was computed on the basis of calibration with polymer-free glucose standard solutions. For the subcutaneous measurement, the bestfitting time shift (13 min) was taken into account. A Regression of the read-out of a sensor applied in the subcutaneous tissue with coordinated capillary blood concentrations (experiment from 27. 10. 1999, compare Table 1 and Fig. 4). Regression coefficient $1.00, r^{2} 0.94$, coefficient of variation 0.17 , mean difference to identity $2.39 \mathrm{mmol} / \mathrm{l}$. B Sensor read out obtained in blood plasma samples in relation to the values of accurate laboratory analysis. Results obtained with two different sensors in 20 blood plasma samples. Regression coefficient $0.80, r^{2} 0.97$, coefficient of variation 0.086 , mean difference to identity $2.75 \mathrm{mmol} / \mathrm{l}$

transient increase after the meals. Subject 4, a person with Type I (insulin-dependent) diabetes mellitus, provoked relatively large changes in the capillary blood sugar concentration by choice of diet and insulin dosage, which were well recorded by the calibrated sensor (Fig. 4). The curve presented followed the capillary blood values with a best-fitting time shift of $13 \mathrm{~min}$. Similar results were obtained in experiments with longer periods of measurement, when hyperglycaemic excursions occurred on the first and second day. As expected from the higher range of variation in the glucose concentrations, the correla- 
Table 1. Summary of authors' self-tests

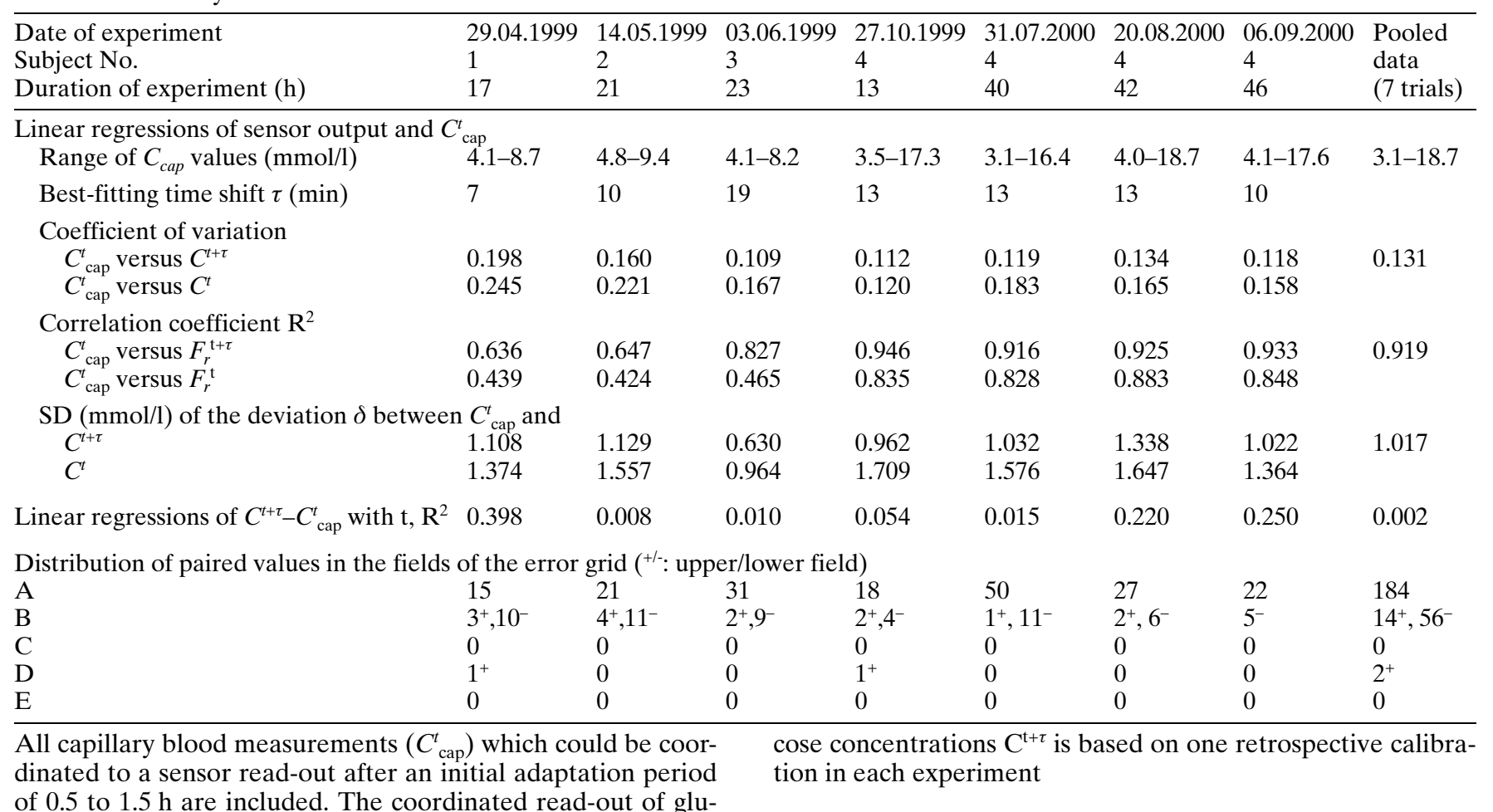

tion coefficients were higher for subject 4 than for subjects 1-3.

Pooled data of all experiments were analysed by the error grid method, which illustrates the therapeutic significance of differences between glucose concentrations obtained by the tested and the reference method [35]. Coincidence is good for $71.8 \%$ and sufficient for $27.3 \%$ of the values (Fig. 5, Table 1).

The delay $\tau$ allowing the best-fit between the sensor read-out and the capillary blood concentration varied between 7 and $19 \mathrm{~min}$. When the read-out was obtained by the standard procedure taking $\tau$ into account, the coefficient of variation obtained with the pooled values $(n=256)$ is 0.13 (Table 1$)$. As a result of retrospective calibration, the linear regression of the capillary blood glucose concentration and the coordinated sensor read-out (Figs. 4 and 5) coincides with the line of identity, and the mean of the differences $(\delta)$ between the sensor read-out and this line [36] was set to zero. These differences varied with standard deviations of 0.63 to $1.34 \mathrm{mmol} / \mathrm{l}$, when the delay was taken into account. Without consideration of $\tau$, the correlation coefficients were lower and the standard deviations were higher. Absolute differences $(|\delta|)$ tended to rise with the glucose concentration (not significant with a probability of $95 \%$, data not shown).

When the deviation between the sensor read-out and the capillary blood concentration $\delta$ was correlated with the measuring time, this did not result in a reproducible trend. The regression coefficient of the pooled values is $-0.003 \mathrm{mmol} \cdot \mathrm{l}^{-1} \cdot \mathrm{h}^{-1}$; $\mathrm{R}^{2}$ values obtained for single experiments are listed in Table 1.

\section{Discussion}

The structure of the dialysis probe we describe protects the cellulose fibre from mechanical forces and allows simple implantation. It should be emphasised that the concept of the relative fluidity [31] has been straightforward for the design of the VAS, as it facilitates calibration and reduces the undesired influence of variations in temperature, air pressure, flow rate and polymer concentrations, all of which strongly affect the absolute measure of viscosity.

The functional properties of the dialysis probes and the measuring, units we tested were well reproducible. The in vitro experiments show that sensitivity, linearity range, response time, reproducibility and measuring stability of the VAS are suitable for glucose monitoring in the physiological system. Our self-testing is only preliminary to the necessary in vivo studies but the results are informative in so far as they show a stable functioning of the VAS in the subcutaneous tissue over 1-2 days. Neither a systematic drift nor a sensitivity change was found within the periods the in vivo application was studied.

The data shown are estimates of capillary blood concentrations obtained by the sensor function in the subcutaneous tissue rather than estimates of the real glucose concentration in the interstitial liquid be- 

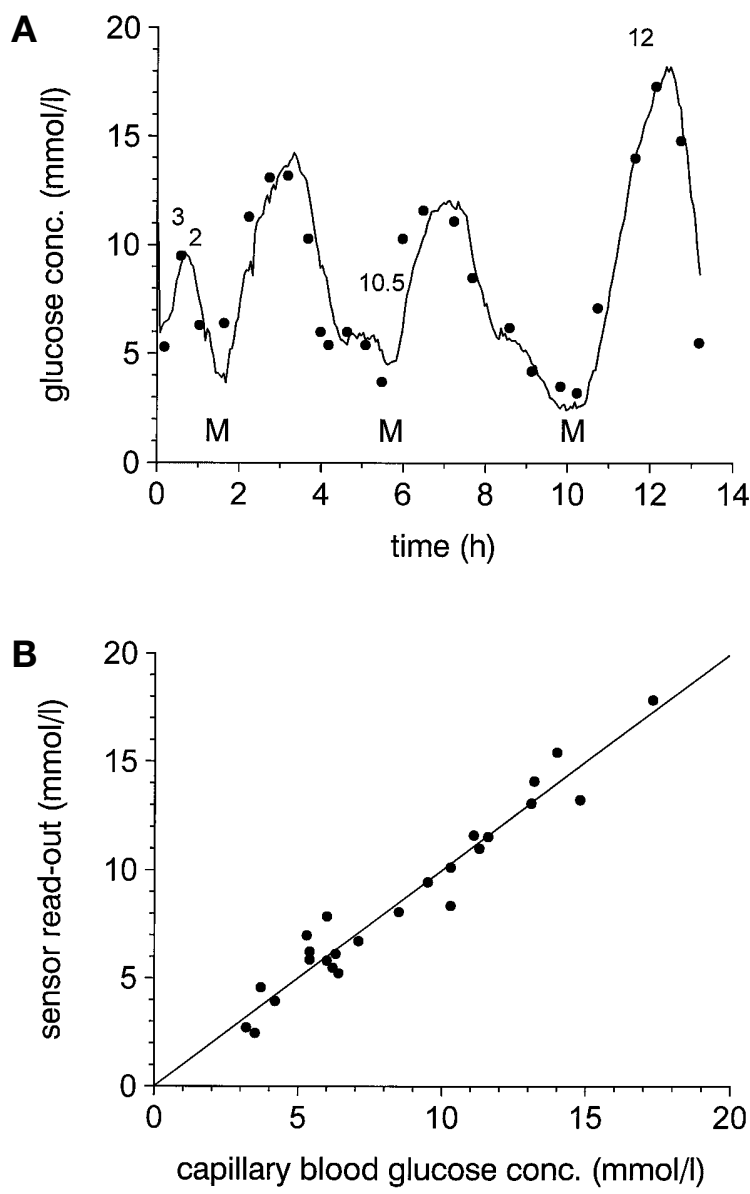

Fig. 4A-B. Recording of the subcutaneous glucose concentration by the VAS (experiment from 27.10.1999). A Time course of capillary blood glucose concentrations (points) and contemporary sensor read-out (line). Sensor read-out is based on one retrospective calibration by capillary blood values taking into account the best-fitting time shift $(\tau=13 \mathrm{~min})$. The subject used the insulin pump H-TRONplus V100 (Disetronic Medical Systems, Sulzbach, Germany) with the insulin preparation H-Tronin 100 (Aventis Pharma Deutschland, Bad Soden, Germany). The pump was programmed to the subjects individual basal rate. Meals are indicated by $\mathrm{M}$, additional amounts of insulin (IU) by numerals. At the 10th hour the subject ate without a preprandial insulin dosage. After reaching a capillary blood value of $15 \mathrm{mmol} / \mathrm{l}$, the subject injected $12 \mathrm{IE}$ of the rapidly absorbed insulin Humalog 100 (Lilly Deutschland, Bad Homburg, Germany). B Correlation of the glucose concentrations read out by the sensor at times $t+13$ min with capillary blood concentration measured at times $t$

cause they are based on calibration by the capillary blood values. The deviations we observed between the sensor read-out and capillary blood glucose concentrations include the combined errors of both measurements and possible fluctuations of the ratio between glucose concentrations in the capillary blood and the interstitial liquid.

We found that calibration with polymer-free glucose solutions is not sufficient for glucose sensing in the blood plasma and subcutaneous tissue because the read-out based on this calibration was lower than

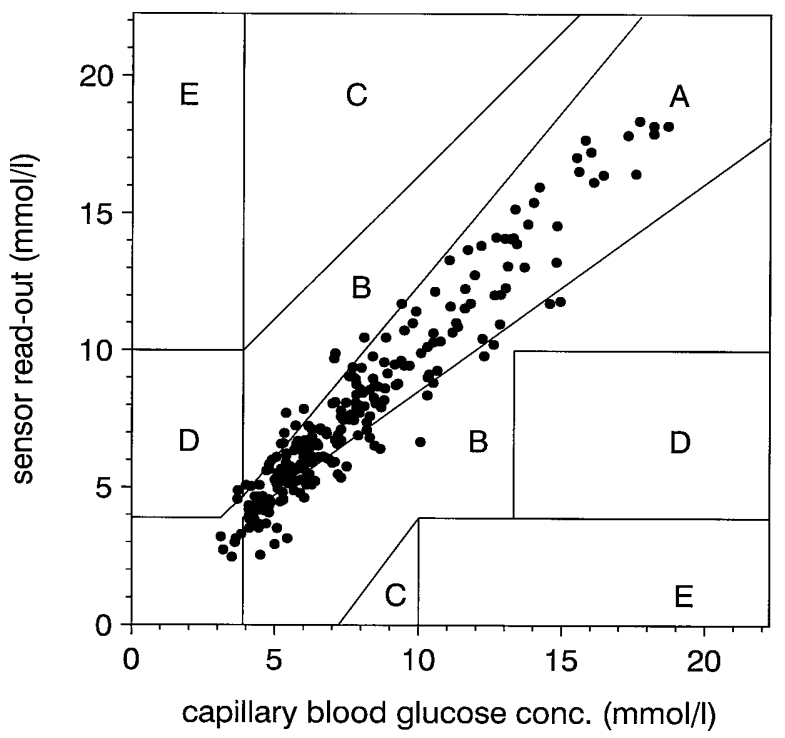

Fig. 5. Error-grid analysis of glucose concentrations read out by the calibrated sensor and coordinated capillary blood values. All values of the experiments summarised in Table 1 $(n=256)$ are included. For each experiment data were processed on the basis of one retrospective calibration using the best-fitting time shift. Fields A-E refer to the potential therapeutic significance of measuring errors. Provided the reference value is correct, appropriate action based on the sensor-readout would be acceptable, if results lay in field A (71.8\%) and B $(27.3 \%)$, but potentially dangerous, if they lay in fields C, $\mathrm{D}$ or $\mathrm{E}(0.9 \%)$

accurate values (plasma) or reasonable estimates (interstitial liquid). At low perfusion rates the colloidosmotic suction of external proteins has a relatively strong effect on the volume flux through the dialysis membrane [37] and this should decrease the readout by increasing the ConA concentration and viscosity in the measuring capillary. Addition of polyethylene glycol to standard glucose solutions in a concentration, which simulates the colloid-osmotic pressure of blood plasma $(\sim 3.4 \mathrm{kPa})$, causes a reduction of the sensor read-out, which is similar to that observed in the blood plasma (unpublished). In future, external calibration will be improved by the use of polymercontaining glucose standard solutions.

Although ConA-based affinity sensors are less sensitive and glucose-specific than GOD sensors, this is not relevant for the application in blood or the subcutaneous tissue, where glucose occurs at a relatively high concentration and is the only sugar species of the dialysate which can interact with the ConA complexes in the fibre lumen. Compared with the enzyme sensors, ConA-based sensors have common advantages: extremely high stability of the glucose receptor protein [30], independence from oxygen pressure or electrode poisons, and the reversible character of the signal transformation process, which enables the equilibration of the sensing polymers with the glucose concentration of the matrix. The latter 
point is especially important, as a constant diffusion resistance for glucose at the sensor surface (a prerequisite of constant sensitivity of the amperometric GOD sensors) seems to be difficult to realise in vivo [13, 16-17].

In the VAS, the proper measuring element, a simple capillary flow resistor, is immediately connected with the dialysis fibre to allow a short response time at flow rates low enough for $100 \%$ recovery [21]. This structure resembles that of a recently described GOD sensor, which combines ultrafiltration or microdialysis at low flow rate with the function of a closely coupled minute GOD-microelectrode [22]. A comparison of the in vivo function of both dialysistype sensors is not yet possible.

The delay in the in vitro sensor response (5-10 min) is larger than expected from the times necessary for sugar diffusion and volume flow through the measuring capillary (1-2 min at a flow rate of $5 \mu \mathrm{l} / \mathrm{h}$ ). The delay is probably due to the elastic volume capacity of the liquid conductor between pressure transducer 2 and the measuring capillary because it is reduced with increasing flow rate and the primary response is a pressure change. This technical delay is possibly a component of the time-shift between changes of the capillary blood glucose concentration and sensor response found in vivo experiments and needs to be reduced by further improvements.

The requirement for a liquid flow carrying ConA, a non-human protein, in relatively high concentration through the dialysis probe must be considered as a technical complication (as in other microdialysis-devices) and is a risk because skin reactions might occur if the dialysis probe leaks. This risk has to be investigated carefully and leaks in the dialysis probe should be excluded rigorously before any clinical study with the VAS. The amount of ConA which can eventually come into contact with the physiological system is extremely small in technically more demanding variants of the VAS because in these variants the ConA solution is restricted to the dialysis fibre segment $[32,33]$.

Comparison of the potential of the VAS with that of currently developed minimally-invasive GOD sensors $[9,18,22]$ would be premature. Irrespective of a possible development of the VAS to a product for routine therapeutic use, the supply of a functioning transdermal sensor with an alternative measuring principle is undoubtedly of great interest for research in the field of wearable glucose sensors because it offers the possibility of an independent in situ reference.

Acknowledgements. The authors would like to express their gratitude to Dr. A. Kage, Humboldt-University Berlin, Medical Faculty (Charitè), Institute of Laboratory Medicine and Pathobiochemistry for providing analysed blood plasma samples.

\section{References}

1. Pfeiffer EF, Thun C, Clemens AH (1974) The artificial beta-cell. A continuous control of blood sugar by external regulation of insulin infusion (glucose-controlled-infusionsystem). Horm Metab Res 6: 339-342

2. Shichiri M, Kawamori R, Hakui N, Askawa N, Yamasaki Y, Abe H (1984) The development of wearable-type artificial endocrine pancreas and its uesfulness in glycemic control of human diabetes mellitus. Biomed Biochim Acta 43: 561-568

3. Sternberg F, Hoss U, Salgado M, Pfeiffer EF (1997) Selfmonitoring of blood glucose compared with continuous tissue glucose measurement. Diabetes Care 20: 1208-1209

4. Jansson PA, Fowelin J, Smith U, Lönnroth P (1988) Characterisation by microdialysis of intercellular glucose level in subcutaneous tissue in humans. Am J Physiol 255: E218-E220

5. Bantle JP, Thomas W (1997) Glucose measurement in patients with diabetes mellitus with dermal interstitial fluid. J Lab Clin Med 130: 436-446

6. Service FJ, O'Brian PC, Wise SD, Ness S, LeBlanc SM (1997) Dermal interstitial glucose as an indicator of ambient glycemia. Diabetes Care 20: 1426-1429

7. Rosdahl, H, Hamrin K, Ungerstedt U, Henriksson J (1998) Metabolite levels in human skeletal muscle and adipose tissue studied with microdialysis at low perfusion flow. Am J Physiol 274: E936-E945

8. Rebrin K, Steil GM, Van Antwerp WP, Mastrototaro JJ (1999) Subcutaneous glucose predicts plasma glucose independent of insulin: implications for continuous monitoring. Am J Physiol 277: E561-E571

9. Tierney MJ, Jayalakshmi Y, Parris NA, Reidy MP, Uhegbu C, Vijayakumar P (1999) Design of a biosensor for continual, transdermal glucose monitoring. Clin Chem 45: $1681-1683$

10. Clark LC, Lyons C (1962) Electrode systems for continuous monitoring in cardiovascular surgery. Ann N W Sci 102: 29-45

11. Updike SJ, Hicks H (1967) The enzyme electrode. Nature 214: 986-988

12. Shichiri M, Kawamora R, Yamasaki Y, Hakui N, Abe H (1982) Wearable artificial endocrine pancreas with needletype glucose sensor. Lancet ii: 1129-1131

13. Müller A, Abel P, Fischer U (1986) Kontinuierliche Messung der Glukosekonzentration im subkutanen Gewebe mit implantierbaren Enzymelektroden. Biomed Biochim Acta 45: 769-777

14. Kerner W, Keck FS, Zier H, Pfeiffer EF (1988) A potentially implantable enzyme electrode for amperometric measurement of glucose. Horm Metab Res [Suppl 20]: 8-13

15. Koudelka M, Rohner-Jeanrenaud F, Terretaz J, BobbioniHarsch E, de Rooij NF, Jeanrenaud B (1991) In-vivo behaviour of hypodermically implanted microfabricated glucose sensors. Biosens Bioelectron 6: 31-36

16. Kerner W, Keck FS, Zier H, Pfeiffer EF (1993) The function of a hydrogen peroxide-detecting electro-enzymatic glucose electrode is markedly impaired in human subcutaneous tissue and plasma. Biosens Bioelectron 8: 475-482

17. Fischer U, Rebrin K, von Woedke T, Abel P (1994) Clinical usefulness of the glucose concentration in the subcutaneous tissue - properties and pitfalls of electrochemical biosensors. Horm Metab Res 26: 515-522

18. Mastrototaro J (1999) The minimed glucose monitoring system (Cgms). J Pediatr Endocrinol Metab 12: 751-758

19. Pfeiffer EF, Meyerhoff C, Bischof F, Keck FS, Kerner W (1993) On-line continuous monitoring of subcutaneous tis- 
sue glucose is feasible by combining portable glucose sensor with microdialysis. Horm Metab Res 25: 121-124

20. Ballerstädt R, Schultz JS (1996) Sensor methods for use with microdialysis and ultrafiltration. Adv Drug Deliv Rev 21: 225-238

21. Kaptein WA, Zwaagstra JJ, Venema K, Korf J (1998) Continuous ultraslow microdialysis and ultrafiltration for subcutaneous sampling as demonstrated by glucose and lactate measurements in rats. Anal Chem 70: 4696-4700

22. Korf J, Rhemrev-Boom R, Tiessen R, Venema K, Jobst G (2000) A miniaturized disposable biosensor system for continuous in vivo monitoring of acetate and glucose. abstract in: Biosensors 2000, The Sixth World Congress on Biosensors, Elsevier, The Boulevard, Langford Lane, Kidlington, Oxford, p 61

23. Schultz J, Mansouri S, Goldstein IJ (1982) Affinity sensor: a new technique for developing implantable sensors for glucose and other metabolites. Diabetes Care 5: 245-253

24. Meadows D, Schultz JS (1988) Fiber-optic biosensors based on fluorescence energy transfer. Talanta 35: 145-150

25. Meadows D, Schultz JS (1993) Design, manufacture and characterization of an optical fiber glucose affinity sensor based on an homogenous fluorescence energy transfer assay system. Anal Chim Acta 280: 21-30

26. Ballerstadt R, Dahn MS, Schultz JS, Lange MP (1993) A homogeneous affinity fluorescence assay system for galactose monitoring. Sensors Actuators B 38-39: 171-175

27. Ballerstadt R, Schultz JS (1997) Competetive-binding assay method based on fluorescence quenching of ligands held in close proximity by a multivalent receptor. Anal Chim Acta 345: 203-212

28. Ehwald R, Ballerstädt R, Dautzenberg H (1996) Viscosimetric affinity assay. Anal Biochem 234: 1-8
29. Beyer PU, Ballerstädt R, Ehwald R (1996) Viskosimetrische Affinitätssensoren. Lebensmittel- \& Biotechnologie 13: $143-146$

30. Ballerstädt R, Ehwald R (1994) Suitability of aqueous dispersions of dextran and Concanavalin A for glucose sensing in different variants of the affinity sensor. Biosens Bioelectron 9: 557-567

31. Beyer U, Ehwald R (2000) Compensation of temperature and Concanavalin A concentration effects for glucose determination by the viscometric affinity assay. Biotechnology Progress 16: 1119-1123

32. Beyer U, Ehwald K-E, Ehwald R, Thomas A (1999) Viskosimetrischer Affinitätssensor. Patent DE 19714087

33. Ehwald R (1996) Mikrosensor zur Bestimmung der Konzentration von Glukose und anderen Analyten in Flüssigkeiten auf der Basis der Affinitätsviskometrie. Offenlegungsschrift DE 19501159 A1

34. Woehlecke H, Ehwald R (1995) Characterization of sizepermeation limits of cell walls and porous separation materials by high-performance size-exclusion chromatography. J Chromatogr A 708: 263-271

35. Clarke WL, Cox DJ, Gonder-Frederick LA, Carter WR, Pohl SL (1987) Evaluating the clinical accuracy of selfblood glucose monitoring systems. Diabetes Care 10: 622-628

36. Bland JM, Altman DG (1986) Statistical methods for assessing agreement between two methods of clinical measurement. Lancet i: $307-310$

37. Rosdahl H, Ungerstedt U, Henriksson J (1997) Microdialysis in human skeletal muscle and adipose tissue at low flow rates is possible if dextran-70 is added to prevent loss of perfusion fluid. Acta Physiol Scand 159: 261-262 